“( 2014 IEEE. Personal use of this material is permitted. Permission from IEEE must be obtained for all other uses, in any current or future media, including

reprinting/republishing this material for advertising or promotional purposes, creating new collective works, for resale or redistribution to servers or lists, or reuse of any copyrighted component of this work in other works." 


\section{A Hybrid System for Skin Lesion Detection: Based on Gabor Wavelet and Support Vector Machine}

\author{
Mohamed Khaled Abu Mahmoud \\ Faculty of Engineering and Information Technolgy \\ University of Technology, Sydney \\ Ultimo NSW 2007 Australia
}

\author{
Adel Al-Jumaily \\ Faculty of Engineering and Information Technology \\ University of Technology, Sydney \\ Ultimo NSW 2007 Australia
}

\begin{abstract}
Severe melanoma is potentially life-threatening. A novel methodology for automatic feature extraction from histopathological images and subsequent classification is presented. The proposed automated system uses a number of features extracted from images of skin lesions through image processing techniques which consisted of a spatially winner and adaptive median filter then applied Gabor filter bank to improve diagnostic accuracy. Histogram equalization to enhance the contrast of the images prior to segmentation is used. Then, a wavelet approach is used to extract the features; more specifically Wavelet Packet Transform (WPT).This article introduces a novel melanoma detection strategy using a hybrid particle swarm - based support vector machine (SVM-WLG - PSO) technique. The extracted features are reduced by using a particle swarm optimization (PSO), this was used to optimize the SVM parameters as a feature selection and finally, the obtained statistics are fed to a support vector machine (SVM) binary classifier to diagnose skin biopsies from patients as either malignant melanoma or benign nevi. The obtained classification accuracies show better performance in comparison to similar approaches for feature extraction. The proposed system is able to achieve one of the best results with classification accuracy of $87.13 \%$, sensitivity of $94.1 \%$ and specificity of $\mathbf{8 0 . 2 2 \%}$.
\end{abstract}

Keywords- Lesion, histo-pathological images, winner, adaptive median filter (AMF), Gabor filter bank, Histogram Equalization, particle swarm optimization (PSO), support vector machine (SVM).

\section{Introduction}

Australia is one of many countries in which skin cancer is simply "wide spread" in comparison to other types of cancer [1] [2]. Researchers have found that Australian melanoma rates are the highest globally at almost four times the rates seen in Canada, the United Kingdom and the United States[3]. Skin cancer costs the health system around $\$ 300$ million Australian dollars annually, the highest cost of all cancers. Melanoma has near $95 \%$ cure rate if detected and treated in its early stages[4]. This study proposes an automated system for discrimination between melanocytic nevi and malignant melanoma. The general approach of developing a Computer Aided Diagnostic system for diagnosis of skin cancer is to find the location of a lesion and also to determine an estimate of the probability of a disease. As mentioned in the literature the digital images are often corrupted during acquisition and transmission[5]. Filters are then very important as pre-processing tools.
Wiener, Gabor and adaptive median filters are used to remove unwanted features like the fine hairs, noise and air bubbles on the skin[6]. Median filter is used to remove noise without blurring edges. In addition these filters have been shown to possess optimal localization properties in both the spatial and frequency domains and thus are well suited for quality segmentation problems[7].

Segmentation is one of the most widely investigated research areas in pathological image analysis. In swarm-based SVM, a particle swarm optimization (PSO) was used to optimize the SVM parameters. A fitness function was defined in the optimization to find a high performance of the melanoma detection, especially in the sensitivity. The Support vector machine (SVM) classier is used widely in bioinformatics, due to its high accuracy, ability to deal with high-dimensional data and in this syntax diverse sources of data[8] [9] [10].

This article introduces a novel melanoma detection strategy using a hybrid particle swarm - based support vector machine (SVM-WLG - PSO) technique. It is possible that the data found from the RBF mapping is more likely to be correctly classified by SVM than from the other kernel functions mappings. Furthermore, SVM-linear could be a special case of SVM-RBF[9] [11], which means that SVM-RBF has more possibilities to obtain a better performance than SVM-linear. The better performance of the SVM based system which employs RBF is confirmed in another application [8] [12].

This paper is organized as follows: its next section describe the computer-aided diagnosing (CAD) which consists of the a) pre-processing, b) segmentation, c) features extraction and selection, and d) the automated procedure using SVM. The followed section presents the result and discussion, and the final section presents conclusions.

\section{Computer-aided design (CAD) for skin lesions}

Automated diagnostic of medical images analyzing digital images has become one of the major medical research areas, and a dynamic area in several applications[13]. It has been shown through research that Melanoma if detected in the early stages improves survival rates. Figure 1 displays the four stages of the CAD system for skin lesion images. 


\section{A. Pre-Processing Stage}

This stage includes image resizing, masking, cropping, hair removal, and conversion from RGB color to intensity grey image. It is meant to facilitate image segmentation by filtering the image and enhancing its important features [5].

\section{- Wiener2 Filter:}

Wiener filtering gives the optimal way of narrowing off the noisy components, so as to give the best reconstruction of the original signal. It can be applied in spatial basis, Fourier basis (frequency components), wavelet basis, etc. Wiener2 is low pass-filters to a gray scale image that has been corrupted by constant power additive noise. The Wiener method is based on statistics estimated from a local neighborhood of each pixel. The additive noise (Gaussian white noise) is assumed to be noise. Wiener filtering is best when applied to the difference between an image and a smoothed image [14] [15] [16].

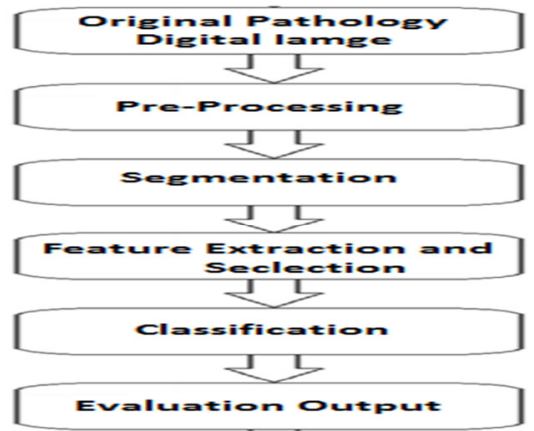

Figure 1. Displays the four stages of the CAD system for skin lesion images.

\section{- $\quad$ Gabor Filter.}

The Gabor filter is basically a Gaussian (with variances $\mathrm{Sx}$ and Sy along $\mathrm{x}$ and $\mathrm{y}$-axes respectively) modulated by a complex sinusoid (with center frequencies $\mathrm{U}$ and $\mathrm{V}$ along $\mathrm{x}$ and $\mathrm{y}$-axes respectively). Gabor filters have been used in many applications, such as quality segmentation, target detection, fractal dimension management, document analysis, edge detection, retina identification, image coding and image representation[17]. A Gabor filter can be viewed as a sinusoidal plane of particular frequency and orientation, modulated by a Gaussian envelope.

Thus the 2-D Gabor filter can be written as in equation 1:

$$
\begin{aligned}
h(x, y) & =e^{-0.5\left(\frac{x^{2}}{\sigma_{x}^{2}}+\frac{x^{2}}{\sigma_{y^{2}}}\right)} e^{-j 2 \omega\left(u_{0} x+v_{0} y\right)} \\
& =g(x, y) e^{-j 2 \pi\left(u_{0} x+v_{0} y\right)}
\end{aligned}
$$

The frequency response of the filter is:

$$
\begin{aligned}
H(u, v) & =G\left(u-u_{0}, v-v_{0}\right) 2 \pi \sigma_{x} \sigma_{y} \\
& =\left[e^{-2 \pi^{2}\left[\left(u-u_{0}\right)^{2} \sigma_{x}^{2}+\left(v-v_{0}\right)^{2} \sigma_{y}{ }^{2}\right]}\right. \\
& =\frac{1}{2 \pi \sigma_{x} \sigma_{y}} \quad e^{-0.5\left[\frac{\left(u-u_{0}\right)^{2}}{\sigma_{u}{ }^{2}}+\frac{\left(v-v_{0}\right)^{2}}{\sigma_{v}{ }^{2}}\right]}
\end{aligned}
$$

$$
\Omega \eta \varepsilon \rho \varepsilon \quad \sigma_{u \frac{1}{2 \pi \sigma_{x}}}, \sigma_{v} \frac{1}{2 \pi \sigma_{y}}
$$

this is equivalent to translating the Gaussian function by $\left(\mathrm{u}_{0}, \mathrm{v}_{0}\right)$ in the frequency domain. Thus the Gabor function can be thought of as being a Gaussian function shifted in frequency to position $\left(\mathrm{u}_{0}, \mathrm{v}_{0}\right)$ i.e. at a distance of $\sqrt{\mathrm{u}_{0}{ }^{2}+\mathrm{v}_{0}{ }^{2}}$ from the origin $\tan ^{-1} \frac{\mathrm{u}_{0}}{\mathrm{v}_{0}}$. In the above equations (1) \& (2), $\square \mathrm{u}_{0} \square \square \mathrm{v}_{0} \square$ are referred to as the Gabor filter spatial central frequency. The parameters $\sigma_{\mathrm{x}}, \sigma_{\mathrm{y}}$ are the standard deviation of the Gaussian envelope along $\mathrm{X}$ and $\mathrm{Y}$ directions and determine the filter bandwidth.

- Median filter:

Median filtering is a nonlinear operation often used in image processing to reduce noise and not preserve edges. The median filter considers each pixel in the image in turn and looks at its nearby neighbors to decide whether or not it is representative of its surroundings [5]. It replaces it with the median of those values. The median is calculated by first sorting all the pixel values from the surrounding neighborhood into numerical order and then replacing the pixel being considered with the middle pixel value. A $3 \times 3$ square neighborhood is used here; larger neighborhoods will produce more severe smoothing[5].

The performance of the filter was evaluated by computing the two criteria, classically used in the literature[18] [19], the mean absolute error (MAE) and the mean square error (MSE), respectively.

\section{B. Image processing}

Segmentation and classification are important steps in the medical image analyses for radiological evaluation or (CAD). One of the early steps in this stage is image enhancement. The purpose of image enhancement methods is to process a picked image for better contrast and visibility of features of interest for visual examination as well as subsequent computer-aided analysis and diagnoses. As described in[14] [20]different medical imaging modalities provide specific characteristics information about internal organs or biological skins. Image contrast and visibility of the features of interest depend on the imaging modality as well as the anatomical regions [7].

The simplest form of intensity transformation function is written as shown in equation 3 :

$$
s=T(r)
$$

where $r$ denotes the intensity of $f$ and $s$ the intensity of $g$, both at any corresponding point $(\mathrm{x}, \mathrm{y})$ in the images.

\section{Histogram Processing.}

Histogram is the estimation of the probability distribution of a particular type of data. The histogram in Figure 2 shows that the image contains only a fraction of the total range of grey levels. Therefore this image has low contrast. We focus on using histograms for image enhancement.

- Histogram Equalization.

This application describes a method of imaging processing that allows medical images to have better contrast. 


\section{Image Segmentation}

Segmentation is one of the most difficult tasks in image processing. Image segmentation methods can be broadly classified into three categories: 1. Edge-based methods, 2. Pixel-based direct classification methods, 3.Region-based methods.

\begin{tabular}{|c|c|c|}
\hline Otiginal true colour image & Imgege after sdiptive median filter & Adjust image intensity \\
\hline Axplies & fos & bis \\
\hline Gry scale imsge & Output image using histogram equalization & Histogram out \\
\hline$\left(\begin{array}{l}8 \\
y^{2}\end{array}\right.$ & & Af \\
\hline
\end{tabular}

Fig. 2. The results showed, original image, image after median filter, gray scale image, histogram out; adjust image intensity, and output image using histogram equalization

- $\quad$ Edge Detection Operations.

The gradient magnitude and directional information from the Sobel horizontal and vertical direction masks can be obtained by convolving the respective Gx and Gy masks with the image. The magnitude $(\mathrm{M})$ of the gradient that can be approximated as the sum of the absolute values of the horizontal and vertical gradient images is obtained by convolving the image with the horizontal and vertical masks, Gx and Gy[21] [22] .

Thresholding.

This has used the gradient to improve the histogram by combining intensity and gradient information for better separation of objects and background, as displayed in figure 3 .
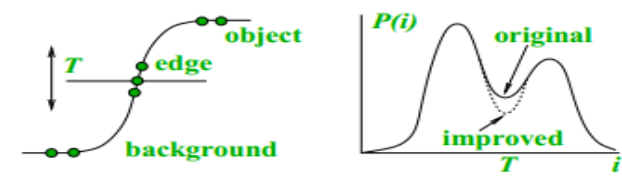

Fig. 3. Selecting a threshold by visually analysing a bimodal histogram. (Principle of histogram peak separation).

\section{- $\quad$ Optimal Global Thresholding}

To determine an optimal global grey value threshold for image segmentation, parametric distribution based methods can be applied to the histogram of an image[23]. Let us assume that the histogram of an image to be segmented has two normal distributions belonging to two respective classes such as background and object. The over-all probability of error in pixel classification using the threshold $\mathrm{T}$ is expressed in equation 4:

$$
\begin{aligned}
E(T) & =P_{2}(T) E_{1}(T)+P_{1}(T) E_{2}(T) \\
E_{1}(T) & =\int_{-\infty}^{T} P_{2}(z) d z \\
E_{2}(T) & =\int_{-\infty}^{T} P_{1}(z) d z
\end{aligned}
$$

where E1 (T) in equation 5 and E2 (T) in equation 6 are the probability of incorrectly classifying a class 1 pixel to class 2 and a class 2 pixels to class 1 respectively.

For image segmentation, the objective is to find an optimal threshold $\mathrm{T}$ that minimizes the overall probability of error in pixel classification. The optimization process requires the parameterization of the probability density distributions and possibility of both classes. These parameters can be determined from a model or set of training images[19].

Let us assume $\sigma \mathrm{i}$ and $\mu \mathrm{i}$ to be the standard deviation and mean of the Gaussian probability density function of the class $i$ ( $\mathrm{i}=1,2$ for two classes) as explained in equation 7 :

$$
\begin{aligned}
& p(z)=P 1 / \sqrt{2 \pi \sigma_{1}} e^{-}\left(z-\mu_{1}\right)^{2} / 2 \sigma_{1}^{2}+\Pi 2 / \sqrt{2 \pi} \sigma_{2} \quad e- \\
& \left(z-\mu_{1}\right)^{2} / 2 \sigma_{2}^{2}
\end{aligned}
$$

The optimal global threshold $\mathrm{T}$ can be determined by finding a general solution that minimizes Equation (4) with the mixture distribution in Equation (7) and thus satisfies the following quadratic expression [2] as in equation 8 :

$$
A T^{2}+B T+C=0 \text {, }
$$

where equations (9) - (11) display the components of equation (10)

$$
\begin{aligned}
& A=\sigma_{1}^{2}-\sigma_{2}^{2} \\
& B=2\left(\mu_{1} \sigma_{2}^{2}-\mu_{2} \sigma_{1}^{2}\right) \\
& C=\sigma_{1}^{2} \mu_{2}^{2}-\sigma_{2}^{2} \mu_{1}^{2}+2 \sigma_{1}^{2} \sigma_{2}^{2} \operatorname{In}\left(\sigma_{2} P_{1} / \sigma_{1} P_{2}\right)
\end{aligned}
$$

If the variances of both classes can be assumed to be equal to $\sigma^{\wedge} 2$, the optimal threshold $\mathrm{T}$ can be determined as in equation 12:

$$
T=\frac{\mu_{1}+\mu_{2}}{2}+\frac{\sigma^{2}}{\mu_{1}-\mu_{2}} \ln \left(\frac{P_{2}}{P_{1}}\right)
$$

It should be noted that in the case of equal probability of classes, the above expression for determining the optimal threshold is simply reduced to the average of the mean values of two classes. In this study were used Edge Detection Operations as the segmentation method.

\section{Feature Extraction}

This paper used six features extracted from histogram, twenty one features were extracted from co-occurrence matrix for each channel and 255 features were extracted from Wavelet Packet Transform (WPT). WPT was used to implement the feature extraction process. Variability appears to be what most separates malignant melanoma from benign nevi, therefore the best approach at feature extraction would retain be to retain as much of the data variability as possible[24]. Wavelet analysis looks at these changes over different scales which should detect whole lesion changes such as texture, color, and local 
changes like granularity. The WPT is a generalized version of the Wavelet Transform: the high-frequency part also splits into a low and a high frequency part, this produce a decomposition tree as shown in the Fig.4.

The WPT provides a high dimensional feature vector thus providing more information about the images. However, the WPT complicates the analysis process as the high dimensionality of the feature vector causes an increase in the learning parameters of the pattern classifier, and the convergence of The learning error deteriorates. Consequently, dimensionality reduction will play an important role before applying the feature vector to the pattern classifier.

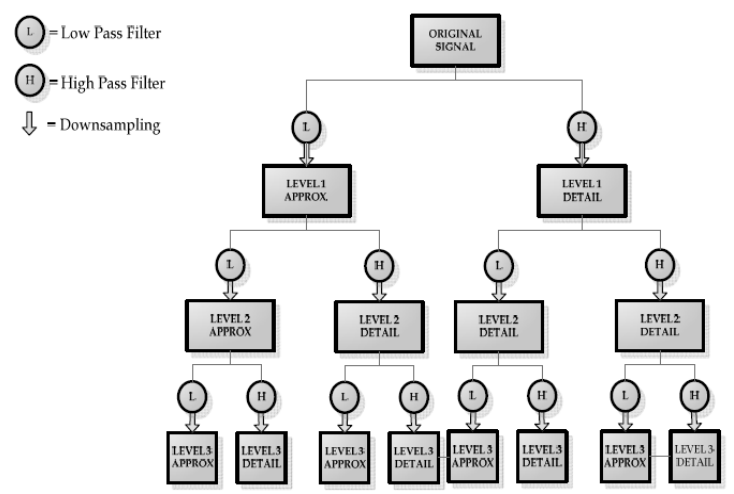

Fig. 4. - A Wavelet Packet decomposition tree

The extracted features were reduced by using a particle swarm optimization (PSO)[25] . This was used to optimize the SVM parameters as a feature selection and finally, the obtained statistics were fed to a support vector machine (SVM) binary classifier to diagnose skin biopsies from patients as either malignant melanoma or benign nevi. The obtained classification accuracies show better performance in comparison to similar approaches for feature extraction.

\section{E. Support Vector Machine based Classification}

The SVM classifier is widely used in bioinformatics, due to its high accuracy, ability to deal with high-dimensional data such as genetic factor expression, and edibility in these special context diverse sources of data[8] [26]. The SVM is used in the classification of histopathology images which is often the final goal in image analysis, particularly in cancer applications. Features derived from segmented nuclei from histopathology are usually a prerequisite to extracting higher level information regarding the state of the disease.

\section{$3 \quad$ Results and Discussion}

Feature Process: In our algorithm we used a 5-fold cross validation test. The LIBSVM[8] [27] is a process library for support vector machines, where the linear, (RBF) Radial Basis Function kernel[8] and Polynomial kernel are each under magnification. The melanoma images that are confirmed by the pathologist are treated as negative images, while the nevus ones are treated as positive images. For instance, the classifying of breast tissue by[18] found $67.1 \%$ accuracy in labeling nuclei as benign or malignant, the classification of histopathology images presented in[28] resulted in $88.7 \%$ accuracy in the diagnosis of lung cancer, $94.9 \%$ accuracy in the typing of malignant mesothelioma, and $80.0 \%$ to $82.9 \%$ accuracy in the prognosis of malignant mesothelioma for Fulgent stained lung sections. Keenan et al [13] reported accuracies of 62.3\%$76.5 \%$ in the grading of H\&E stained cervical tissue. Skin cancer is a fast developing disease of modern society, reaching $20 \%$ [13] increase of diagnosed cases every year. As reported in[13], only experts arrive at $90 \%$ sensitivity and $59 \%$ specificity in skin lesion diagnosis, while for less trained doctors, these figures shown a significant drop to around 62- 63\% for general practitioners, as mentioned on Table 1.

TABLE I. DESCRIBED SENSITIVITY AND SPECIFICITY

\begin{tabular}{|l|l|l|}
\hline & Sensitivity & Specificity \\
\hline Experts & $90 \%$ & $\mathbf{5 9} \%$ \\
\hline Dermatologists & $\mathbf{8 1} \%$ & $\mathbf{6 0} \%$ \\
\hline Trainees & $\mathbf{8 5} \%$ & $\mathbf{3 6} \%$ \\
\hline General practitioners & $\mathbf{6 2} \%$ & $\mathbf{6 3} \%$ \\
\hline
\end{tabular}

The presented work has been implemented using MATLAB R2013b, and tested using 79 images ( 29 benign images and 50 melanoma images). The images are sampled and split into training set (mixed 64 images) and test set (mixed 15 images). These 79 images were fed into the proposed SVM network. The performance of the algorithm was evaluated by computing the percentages of Sensitivity (SE) equation (13), Specificity (SP) equation (14) and Accuracy (AC) equation (15): the respective definitions are as follows:

$$
\begin{array}{r}
S E=\frac{T P}{(T P+F N)} \times 100 \\
S P=\frac{T N}{(T N+F P)} \times 100 \\
A C=\frac{(T P+F N)}{(T N+T P+F N+F P)}
\end{array}
$$

where TP is the number of true positives (expects malignant as malignant), TN is the number of true negatives (expects benign as benign), $\mathrm{FN}$ is the number of false negatives (expects malignant as benign), and FP is the number of false positives (predicts benign as malignant). Since it is interesting to estimate the performance of classifier based on the classification of benign and malignant skin cell nuclei, sensitivity, specificity and accuracy of prediction have been calculated according to the above equations for all of the testing data. Table 2 shows the average resulting $\mathrm{SE}, \mathrm{SP}$ and $\mathrm{AC}$ of 5 fold validation of the proposed networks by using LIBSVM. This study used RBF kernel with gamma $=0.0084732$ and $\mathrm{C}=3525.0051$. We did many trials to improve our experimental results; table 3 shows the results without using (SFS) technique. These low results can be compared with our proposed results displayed in table 2 by using (SFS) sequential feature selection technique.

TABLE 2. THE RESULT AFTER TRAINING OF THE (SVM) NETWORK. WITH USING (SVM+WLG+PSO)

\begin{tabular}{|l|l|l|l}
\hline No of images & Sensitivity & Specificity & Accuracy \\
\hline
\end{tabular}


79

94.1

80.2

87.1

TABLE 3. THE RESULT AFTER TRAINING OF THE (SVM) NETWORK, WITHOUT USING (SFS)TECHNIQUE.

\begin{tabular}{|c|l|l|l|}
\hline No of images & Sensitivity & Specificity & Accuracy \\
\hline 79 & 83.6 & 70.7 & 77.4 \\
\hline
\end{tabular}

4

Conclusion.

In this paper, SVM has been implemented for classification of benign from malignant skin tumor. 79 images sampled from microscopic slides of skin biopsy have been used in the current work. MATLAB software is used to implement the proposed work; these features were carried out to generate training and testing of the proposed SVM. The present work is a new application based on histo-pathological images of skin lesions that required finding out new features getting the reduced numbers of features and getting better accuracy. It was required to modify many of the mentioned techniques to make them work for such an application. The higher accuracy of diagnosis of the proposed work is calculated and displayed. The results show that using the (SVM+WLG+PSO) technique produces one of the best results compared with expertise / physicians results displayed in table 1 . The obtained accuracy of the system is $87.1 \%$ whereas the sensitivity and specificity were found to equal $94.1 \%$ and $80.2 \%$ respectively. By comparing the results in tables 1 and 2, it can be seen that the proposed system produced more accurate classification results than physicians did. This paper concludes that there are some possible factors to improve the accuracy of detecting malignant melanoma by having a higher number of images for training of the SVM network. Future work directions will be to use a hybrid approach of genetic algorithms and another different method to improve feature extraction and feature selection. Moreover, we are planning to use different classification techniques such as Neuron-Fuzzy algorithms to improve the accuracy.

A. B.O.S., "Causes of death 2010," Commonwealth of Australia, 2012.

A. I. o. H. a. Welfare, "Cancer in Australia: an overview," A. I. o. H. a. Welfare, Ed., ed. Canberra, Australia: Australian Institute of Health and Welfare, 2006.

P. Baade and M. Coory, "Trends in melanoma mortality in Australia: 1950-2002 and their implications for melanoma control," Australian and New Zealand Journal of Public Health, vol. 29, pp. 383-386, 2007.

AIHW, "Cancer in Australia 2001," Australian Institute of Health and Welfare, Canberra, Australia Cat. No. CAN 23.), 2004.

M. Jayamanmadharao, M. S. Ramanaidu, and K. Reddy, "Impulse Noise Removal from Digital Images- A Computational Hybrid Approach," Global Journal of Computer Science and Technology Graphics \& Vision, vol. 13, 2013.

F. Robert-Inacio, Dinet, E., "An adaptive median filter for colour image processing," Proc. 3rd CGIV, pp. 205-210, 2006.

A. K. Jain, "Fundamentals of Digital Image Processing," Englewood Cliffs, 1989.

B. Scholkopf, Tsuda, K., Vert, J.P., "kernel Methods in computational biology," MIT Press, 2004.

V. Kecman, Learning and Soft Computing: Support Vector Machines, Neural Networks, and Fuzzy Logic Models (Complex Adaptive Systems). Cambridge, MA, 2001: The MIT Press, 2001.

L. P. Wang and X. J. Fu, Data Mining with Computational Intelligence. Berlin: Springer, 2006.

A. Sloin and D. Burshtein, "Support Vector Machine Training for Improved Hidden Markov Modeling," Signal Processing, IEEE Transactions, vol. 56, pp. 172-188, Jan. 20082008.

F. Chu and L. P. Wang, "Applications of support vector machines to cancer classification with microarray data," International Journal of Neural Systems, vol. 15, pp. 475-484, 2005.

R. H., C. H. Chan, and M. Nikolova, "SaIt-and-Pepper Noise Removal by Median-Type Noise Detectors and Detail-Preserving Regularization," IEEE TRANSACTIONS ON IMAGE PROCESSING, vol. I4, 2005.

A. Criminisi, P. Perez, and K. Toyama, "Region filling and object removal by exemplar-based image inpainting," IEEE Transactions on Image Processing, vol. 13, pp. 1200-1212, 2004.

M. OpenCourseWare and http://ocw.mit.edu, "Introduction to Communication, Control, and Signal Processing For Wiener Filtering," S. 2010S, Ed., ed, 2010.

W. Page. (2014). Wiener Filtering and Image Processing. Available: https://www.clear.rice.edu/elec431/projects95/lords/wiener.html

J. Pavlovicova, M. Oravec, and M. Osadský. (2010, An application of Gabor filters for texture classification ELMAR, 2010 PROCEEDINGS 23-26. QinzLi, "Dark line detection with line width extraction," in 15th IEEE International Conference on Image Processing, 2008. ICIP 2008, ed, 2008.

R. M. Hodgson, Bailey, D.G., Naylor, M.J., Ng, A.L.M., McNeill, S.J., "Properties, implementations and applications of rank filters," image and vision computing, vol. 3, pp. 1-14, 1985.

M. R. Sweet, "adaptive and recursive median filtering ".

A. Merritt, M. Mehta, and A. Warke, "Parallel Edge Detection," 2011.

Q. Abbas, " Lesion border detection in dermoscopy images using dynamic programming," Skin Research and Technology, vol. 17, pp. 91-100, 2011.

S. Wu and A. Amin, Eds., Automatic Thresholding of Gray-level Using Multi-stage Approach (Proceedings of the Seventh International Conference on Document Analysis and Recognition (ICDAR 2003). IEEE computer Socity, 2003, p.^pp. Pages.

J. Sikorski, "Identification of malignant melanoma by wavelet analysis," in Student/Faculty Research Day, CSIS, Pace University., 2004. 
[25] Y. del Valle, G. K. Venayagamoorthy, S. Mohagheghi, J.-C. Hernandez, and R. G. Harley, "Particle Swarm Optimization: Basic Concepts, Variants and Applications in Power Systems," IEEE TRANSACTIONS ON EVOLUTIONARY COMPUTATION, vol. 12, pp. 171-195, 2008.

[26] L. P. Wang, B. Liu, and C. R. Wan, "Classification using support vector machines with grading resolution," IEEE International Conference on Granular Computing, vol. 2, pp. 666-670, 2005.

[27] A. H. Khandoker, M. Palaniswami, and C. K. Karmakar, "Support Vector Machine for Automated Recognition of Obstructive Sleep Apnea Syndrome From ECG Recordings," IEEE Trans. Information Technology in Biomedicine, vol. 13, pp. 37-48, 2009.

[28] J. C. Caicedo, A. Cruz, and A. Gonzalez, "histopathology image classification using Bag of Features and Kernel Functions," Bioingenium Research Group. 